\title{
MARKUSCHEVICH BASES AND SEMIREFLEXIVITY ${ }^{1}$
}

\author{
PAUL CARLTON WOODS ${ }^{2}$
}

\begin{abstract}
It is shown that a locally convex space $E$ with a countable Markuschevich basis is semireflexive if each countable $M$-basis for $E$ is shrinking or if each countable $M$-basis for $E$ is boundedly complete.
\end{abstract}

1. Introduction. Since R. C. James [2] established a characterization of reflexivity in a Banach space with a basis in terms of certain properties of that basis, much work in this area has appeared. One approach has been to extend the characterization to spaces more general than Banach spaces. J. R. Retherford [5], Ju. B. Tumarkin [7], and T. A. Cook [1] are examples. Cook's result is the most general; a locally convex space with a Schauder basis $\left\{x_{n}\right\}_{n=1}^{\infty}$ is semireflexive if and only if $\left\{x_{n}\right\}_{n=1}^{\infty}$ is both shrinking and boundedly complete. W. B. Johnson [3] has introduced the concepts of shrinking and boundedly complete Markuschevich bases. He proved that a locally convex space with a $M$-basis $\left\{x_{\lambda}, f_{\lambda}\right\}_{\lambda \in \Lambda}$ is semireflexive if and only if $\left\{x_{\lambda}, f_{\lambda}\right\}_{\lambda \in \Lambda}$ is both shrinking and boundedly complete. Cook's result may be deduced from Johnson's.

I. Singer [6], M. Zippin [8], and N. J. Kalton [4] have used a different approach. Kalton, in generalizing Singer's work in Banach spaces with bases, has proved that a sequentially complete locally convex space $E$ with a Schauder basis is semireflexive if and only if every Schauder basic sequence in $E$ is shrinking or if and only if every Schauder basic sequence in $E$ is boundedly complete. Zippin's result for Banach spaces with bases is also contained in a theorem of Kalton; a complete barrelled space $E$ with a normalized Schauder basis is reflexive if each normalized Schauder basis for $E$ is shrinking or if each normalized Schauder basis for $E$ is boundedly complete.

Received by the editors March 23, 1972.

AMS (MOS) subject classifications (1970). Primary 46A01, 46A05, 46A25; Secondary 46A07, 46A35, 46B10, 46B15.

Key words and phrases. Locally convex space, barrelled space, semireflexivity, bi-. orthogonal system, Markuschevich basis, Schauder basis.

${ }^{1}$ The material in this paper is included in the author's Florida State University dissertation which was written under the supervision of Professor Charles W. McArthur.

${ }^{2}$ The author was supported as an NDEA Fellow.

(c) American Mathematical Society 1973 
The theorems of this paper establish Kalton's characterization for semireflexivity in locally convex spaces with countable Markuschevich bases.

2. Preliminaries. A biorthogonal system $\left\{x_{\lambda}, f_{\lambda}\right\}_{\lambda \in \Lambda}$ for a topological vector space $E$ (each $f_{\lambda} \in E^{\prime}$, the topological dual of $E$ ) is a Markuschevich basis (M-basis) for $E$ if $\left\{x_{\lambda}\right\}_{\lambda \in \Lambda}$ is fundamental in $E$ (i.e., $\left.E=\left[x_{\lambda}\right]\right)$ and if $\left\{f_{\lambda}\right\}_{\lambda \in \Lambda}$ is total over $E$. The $M$-basis $\left\{x_{\lambda}, f_{\lambda}\right\}_{\lambda \in \Lambda}$ is shrinking if $\left\{f_{\lambda}\right\}_{\lambda \in \Lambda}$ is strongly fundamental in $E^{\prime}$. Following Johnson [3], a $M$-basis $\left\{x_{\lambda}, f_{\lambda}\right\}_{\lambda \in \Lambda}$ is boundedly complete if whenever $\left\{Y_{d}: d \in D\right\}$ is a bounded net in $E$ such that $\lim _{d} f_{\lambda}\left(Y_{d}\right)$ exists for each $\lambda \in \Lambda$ there is an $x \in E$ such that $f_{\lambda}(x)=$ $\lim _{d} f_{\lambda}\left(Y_{d}\right)$ for each $\lambda \in \Lambda$. The following existence theorem is used in $\S 3$; for a proof of this theorem see [3].

EXISTENCE THEOREM. Let E be a separable topological vector space. If $Y$ is a strongly closed, strongly separable subspace of $E^{\prime}$ which is total over $E$, then there is a countable $M$-basis $\left\{x_{n}, f_{n}\right\}_{n=1}^{\infty}$ for $E$ with $Y=\left[f_{n}\right]$.

\section{Characterizations of semireflexivity.}

THEOREM 1. Let E be a locally convex space with a countable M-basis. If each countable $M$-basis for $E$ is shrinking, then $E$ is semireflexive.

Proof. If $E$ is not semireflexive, there is a $\phi \in E^{\prime \prime}$ which is not weak star continuous. If $Y$ is the kernel of $\phi, Y$ is a strongly closed subspace of codimension one. Thus, $Y$ is strongly complemented in $E^{\prime}$. Also, since $\phi$ is not weak star continuous, $Y$ is weak star dense in $E^{\prime}$ and hence total over $E$. Since $E$ has a countable $M$-basis and since this $M$-basis is shrinking, $E^{\prime}$ is strongly separable. Hence, the complemented subspace $Y$ is strongly separable. By the existence theorem there is a countable $M$-basis $\left\{x_{n}, f_{n}\right\}_{n=1}^{\infty}$ for $E$ with $Y=\left[f_{n}\right]$. But $\left\{x_{n}, f_{n}\right\}_{n=1}^{\infty}$ must be shrinking; hence, $E^{\prime}=\left[f_{n}\right]$. Thus, $E^{\prime}=Y$. This contradiction yields the desired conclusion.

THEOREM 2. Let $E$ be a locally convex space with a countable M-basis $\left\{x_{n}, f_{n}\right\}_{n=1}^{\infty}$. If each countable $M$-basis for $E$ is boundedly complete, then $E$ is semireflexive.

Proof. We show that $E$ is weakly quasi-complete. Let $\left\{Y_{d}: d \in D\right\}$ be a bounded net in $E$ which is weakly Cauchy. Then $\lim _{d} f_{n}\left(Y_{d}\right)$ exists, $n=1,2, \cdots$. Since $\left\{x_{n}, f_{n}\right\}_{n=1}^{\infty}$ is boundedly complete, there is an $x$ in $E$ such that $f_{n}(x)=\lim _{d} f_{n}\left(Y_{d}\right), n=1,2, \cdots$. It suffices to show that $\left\{Y_{d}: d \in D\right\}$ converges weakly to $x$. Let $f_{0}$ be an arbitrary element of $E^{\prime}$. Then $\left[f_{k}\right]_{k=0}^{\infty}$ is a strongly closed, strongly separable subspace of $E^{\prime}$ which is total over $E$. By the existence theorem there is a countable $M$-basis 
$\left\{y_{n}, g_{n}\right\}_{n=1}^{\infty}$ for $E$ with $\left[f_{k}\right]_{k=0}^{\infty}=\left[g_{n}\right]_{n 1=}^{\infty}$. Since $\left\{y_{n}, g_{n}\right\}_{n=1}^{\infty}$ is boundedly complete, there is a $y$ in $E$ such that $g_{n}(y)=\lim _{d} g_{n}\left(Y_{d}\right), n=1,2, \cdots$.

Claim. $f_{k}(y)=\lim _{d} f_{k}\left(Y_{d}\right), k=0,1,2, \cdots$.

Proof of Claim. Since $B=\left\{Y_{d}: d \in D\right\} \cup\{y\}$ is a bounded subset of $E$, for a fixed $k, k=0,1,2, \cdots$, and $\varepsilon>0$ there is a finite linear combination $g$ of $\left\{g_{n}\right\}_{n=1}^{\infty}$ with $f_{k}-g \in(\varepsilon / 3) B^{0}$. Since $g(y)=\lim _{d} g\left(Y_{d}\right)$, there is a $d_{0}$ in $D$ such that $d \geqq d_{0}$ implies $\left|g\left(Y_{d}\right)-g(y)\right|<\varepsilon / 3$. Hence, for $d \geqq d_{0}$ we have

$$
\begin{aligned}
\left|f_{k}\left(Y_{d}\right)-f_{k}(y)\right| \leqq & \left|f_{k}\left(Y_{d}\right)-g\left(Y_{d}\right)\right|+\left|g\left(Y_{d}\right)-g(y)\right| \\
& +\left|g(y)-f_{k}(y)\right|<\varepsilon,
\end{aligned}
$$

and the claim is proved.

Now

$$
f_{n}(x)=\lim _{d} f_{n}\left(Y_{d}\right)=f_{n}(y), \quad n=1,2, \cdots,
$$

and $\left\{f_{n}\right\}_{n=1}^{\infty}$ is total over $E$. Thus, $x=y$. Hence, $f_{0}(x)=f_{0}(y)=\lim _{d} f_{0}\left(Y_{d}\right)$.

The previously mentioned results of Johnson and Kalton now yield two corollaries.

COROLlary 1. Let $E$ be a sequentially complete locally convex space with a Schauder basis. Then the following are equivalent:

(i) $E$ is semireflexive.

(ii) Each Schauder basic sequence in $E$ is shrinking.

(iii) Each countable M-basis for $E$ is shrinking.

(iv) Each Schauder basic sequence in $E$ is boundedly complete.

(v) Each countable M-basis for $E$ is boundedly complete.

COROLlARY 2. Let $E$ be a complete barrelled space with a normalized Schauder basis. Then the following are equivalent:

(i) $E$ is reflexive.

(ii) Each normalized Schauder basis for $E$ is shrinking.

(iii) Each countable $M$-basis for $E$ is shrinking.

(iv) Each normalized Schauder basis for $E$ is boundedly complete.

(v) Each countable M-basis for $E$ is boundedly complete.

\section{REFERENCES}

1. T. A. Cook, Schauder decompositions and semi-reflexive spaces, Math. Ann. 182 (1969), 232-235. MR 40 \#3255.

2. R. C. James, Bases and Reflexivity of Banach spaces, Ann. of Math. (2) 52 (1950), 518-527. MR 12, 616.

3. W. B. Johnson, Markuschevich bases and duality theory, Trans. Amer. Math. Soc. 149 (1970), 171-177. MR 41 \#5927.

4. N. J. Kalton, Schauder bases and reflexivity, Studia Math. 38 (1970), 255-266. MR 43 \#2457. 
5. J. R. Retherford, Bases, basic sequences and reflexivity of linear topological spaces, Math. Ann. 164 (1966), 280-285. MR 33 \#6351.

6. I. Singer, Basic sequences and reflexivity of Banach spaces, Studia Math. 21 (1961/62), 351-369. MR 26 \#4155.

7. Ju. B. Tumarkin, Locally convex spaces with basis, Dokl. Akad. Nauk SSSR 195 (1970), 1278-1281 = Soviet Math. Dokl. 11 (1970), 1672-1675. MR 42 \#6575.

8. M. Zippin, A remark on bases and reflexivity in Banach spaces, Israel J. Math. 6 (1968), 74-79. MR 38 \#4972.

Department of Mathematics, Florida State University, Tallahassee, Florida 32306

Current address: Division of Sciences, Auburn University at Montgomery, Montgomery, Alabama 36109 